\title{
Mitigation of defocusing by statics and near-surface velocity errors by interferometric least-squares migration with a reference datum
}

\author{
Mrinal Sinha ${ }^{1}$ and Gerard T. Schuster ${ }^{1}$
}

\begin{abstract}
Imaging seismic data with an erroneous migration velocity can lead to defocused migration images. To mitigate this problem, we first choose a reference reflector whose topography is well-known from the well logs, for example. Reflections from this reference layer are correlated with the traces associated with reflections from deeper interfaces to get crosscorrelograms. Interferometric least-squares migration (ILSM) is then used to get the migration image that maximizes the crosscorrelation between the observed and the predicted crosscorrelograms. Deeper reference reflectors are used to image deeper parts of the subsurface with a greater accuracy. Results on synthetic and field data show that defocusing caused by velocity errors is largely suppressed by ILSM. We have also determined that ILSM can be used for 4D surveys in which environmental conditions and acquisition parameters are significantly different from one survey to the next. The limitations of ILSM are that it requires prior knowledge of a reference reflector in the subsurface and the velocity model below the reference reflector should be accurate.
\end{abstract}

\section{INTRODUCTION}

An erroneous velocity model at shallow depths can severely defocus the migration image at intermediate and deep depths. These velocity errors may arise due to a complex overburden or low-velocity anomalies in the shallow subsurface. To mitigate the shallow velocity problem, static corrections are applied to the traces, or the data are redatumed below the problem areas. Many redatuming techniques (Berryhill, 1986; Yilmaz and Lucas, 1986; Bevc, 1997) use the wave equation and require an accurate velocity model above the reference datum.

The other class of redatuming methods uses interferometric principles for moving sources or receivers closer to the target of interest, and therefore, do not require a known velocity model. Bakulin and Calvert (2004) propose VSP data as natural Green's functions to redatum the data to a datum below the complex overburden. Hornby et al. (2006), Hornby and Yu (2007), and Xiao and Schuster (2006) use interferometric redatuming for migrating VSP using the local velocity around the well. Xiao and Schuster (2009) use VSP data as natural Green's function for redatuming CDP data below complex overburden. For surface seismic data, Henley (2012) uses trace crosscorrelations to extract surface functions and deconvolved them from the traces, thereby accounting for distortions in the timing and waveform.

Zhou et al. (2006) introduce the concept of interferometric migration to mitigate the defocusing due to the statics in the data. In this method, they shift the data by the traveltimes of the picked reference reflections. This time shift can also be automatically computed by crosscorrelating the trace windowed around the reference reflection with the original trace. This procedure is carried out for all the traces to theoretically cancel out the phase associated with the common raypaths above the reference interface for small sourcereceiver offsets. It also approximately redatums the data to the reference interface without a known velocity model. One problem with this method is that the correlated traces can lead to artifacts in the migration image and the reference reflections must be carefully windowed. To mitigate the correlation artifacts in the final migration image, we extend the interferometric migration method of Zhou et al. (2006) to a least-squares migration scheme, in which the final migration image is the one that minimizes an interferometric crosscorrelation objective function. Here, the objective function is formed by zero-lag correlation of the recorded and simulated crosscorrelograms.

This paper is organized into four sections. After the introduction, the second section describes the theory of interferometric least-

\footnotetext{
Manuscript received by the Editor 16 November 2015; revised manuscript received 21 February 2016; published online 23 May 2016.

${ }^{1}$ King Abdullah University of Science and Technology, Department of Earth Science and Engineering, Thuwal, Saudi Arabia. E-mail: mrinal.sinha@kaust. edu.sa; gerard.schuster@kaust.edu.sa.

(C) 2016 Society of Exploration Geophysicists. All rights reserved.
} 
squares migration (ILSM). The objective function is defined, and the associated gradient is derived for use in a conjugate gradient method. Numerical results on synthetic and field data are presented in the next section. We also show an example in which ILSM can be used to suppress the influence of environmental variations in repeat surveys. The conclusions are in the last section.

\section{THEORY OF ILSM}

Let the predicted trace in the frequency domain be denoted by $D(\mathbf{g} \mid \mathbf{s})$ for a source at $\mathbf{s}$ and geophone at $\mathbf{g}$. Let $D(\mathbf{g} \mid \mathbf{s})_{\text {ref }}$ denote the trace that is windowed around a reference reflection event as illustrated in Figure 1. To estimate the crosscorrelogram $\Phi(\mathbf{g} \mid \mathbf{s})$, the windowed reference reflections in the predicted data are temporally crosscorrelated with the predicted data. In the frequency domain, crosscorrelation is equivalent to the conjugated product of spectra

$$
\Phi(\mathbf{g} \mid \mathbf{s})=D(\mathbf{g} \mid \mathbf{s}) D^{*}(\mathbf{g} \mid \mathbf{s})_{\text {ref }},
$$

which removes the two-way propagation time from the surface to the reflector for near-offset traces. For example, denote the two-way propagation time to the reference interface as $\tau_{\mathbf{S x}_{\text {ref }}}+\tau_{\mathbf{x}_{\text {ref }} \mathbf{g}}$ so that $D(\mathbf{g} \mid \mathbf{s})_{\text {ref }}=e^{i \omega\left(\tau_{\mathbf{s x}_{\text {ref }}}+\tau_{\mathbf{x}_{\text {ref }} \mathbf{g}}\right)}$. If the reflection data from a deeper interface are given as $D(\mathbf{g} \mid \mathbf{s})=e^{\left(\tau_{\mathbf{s x}_{\mathbf{0}}}+\tau_{\mathbf{x}_{\mathbf{0}} \mathbf{g}}\right)}$ then $D(\mathbf{g} \mid \mathbf{s}) D(\mathbf{g} \mid \mathbf{s})_{\mathrm{ref}}^{*}=$

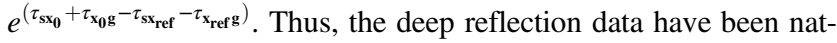
urally redatumed to the reference reflector without knowing the velocity model. However, the implicit assumption is that the reflection rays for the reference reflection coincide with a portion of the rays associated with the deep reflection. Similarly, the observed crosscorrelogram $\tilde{\Phi}(\mathbf{g} \mid \mathbf{s})$ can be obtained by the crosscorrelation of recorded traces with the observed reference reflection traces. The goal is to find the reflectivity model that maximizes the normalized dot product of the observed and predicted crosscorrelograms. This can be written as (Routh et al., 2011; Zhang et al., 2013; Dutta et al., 2014)

$$
\epsilon=-\sum_{\omega} \sum_{s} \sum_{g} \frac{\Phi(\mathbf{g} \mid \mathbf{s})^{*}}{\|\Phi(\mathbf{g} \mid \mathbf{s})\|} \frac{\tilde{\Phi}(\mathbf{g} \mid \mathbf{s})}{\|\tilde{\Phi}(\mathbf{g} \mid \mathbf{s})\|},
$$

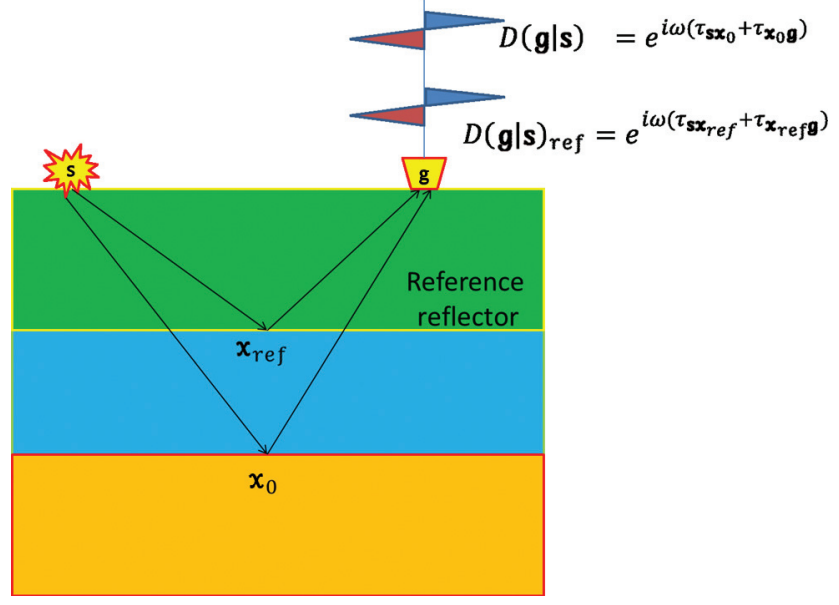

Figure 1. A crosscorrelogram is obtained by crosscorrelating a recorded trace with the same trace windowed around the reference reflection. where we call this an interferometric objective function. The gradient of equation 2 with respect to the perturbation in slowness $s(\mathbf{x})$ is

$$
\begin{gathered}
\frac{\partial \epsilon}{\partial s(\mathbf{x})}=-\sum_{\omega} \sum_{s} \sum_{g} \frac{1}{\|\Phi(\mathbf{g} \mid \mathbf{s})\|} \frac{\partial \Phi(\mathbf{g} \mid \mathbf{s})}{\partial s(\mathbf{x})} \\
\times\left[w(\mathbf{g} \mid \mathbf{s}) \frac{\Phi(\mathbf{g} \mid \mathbf{s})}{\|\Phi(\mathbf{g} \mid \mathbf{s})\|}-\frac{\tilde{\Phi}(\mathbf{g} \mid \mathbf{s})}{\|\tilde{\Phi}(\mathbf{g} \mid \mathbf{s})\|}\right],
\end{gathered}
$$

where $w(\mathbf{g} \mid \mathbf{s})$ is the weight given by $w(\mathbf{g} \mid \mathbf{s})=(\Phi(\mathbf{g} \mid \mathbf{s}) /\|\Phi(\mathbf{g} \mid \mathbf{s})\|)$. $(\tilde{\Phi}(\mathbf{g} \mid \mathbf{s}) /\|\tilde{\Phi}(\mathbf{g} \mid \mathbf{s})\|)$, which emphasizes the phase mismatch between the observed and the predicted crosscorrelograms. If two crosscorrelograms match perfectly, then $w(\mathbf{g} \mid \mathbf{s})=1$.

Substituting the expression for predicted crosscorrelograms in equation 1 into equation 3 gives

$$
\begin{aligned}
\frac{\partial \epsilon}{\partial s(\mathbf{x})}=- & \overbrace{\frac{\partial D^{*}(\mathbf{g} \mid \mathbf{s})}{\partial s(\mathbf{x})}}^{\text {Migration kernel }} \\
\times & \overbrace{\frac{1}{\|\Phi(\mathbf{g} \mid \mathbf{s})\|} D(\mathbf{g} \mid \mathbf{s})_{\operatorname{ref}}\left[w(\mathbf{g} \mid \mathbf{s}) \frac{\Phi(\mathbf{g} \mid \mathbf{s})}{\|\Phi(\mathbf{g} \mid \mathbf{s})\|}-\frac{\tilde{\Phi}(\mathbf{g} \mid \mathbf{s})}{\|\tilde{\Phi}(\mathbf{g} \mid \mathbf{s})\|}\right]}^{\text {Weighted crosscorrelogram residual }}
\end{aligned}
$$

where the Fréchet derivative $\partial D(\mathbf{g} \mid \mathbf{s}) / \partial s(\mathbf{x})$ is given by Luo and Schuster (1991) as

$$
\frac{\partial D(\mathbf{g} \mid \mathbf{s})}{\partial s(\mathbf{x})}=2 \omega^{2} s(\mathbf{x}) G(\mathbf{g} \mid \mathbf{x}) G(\mathbf{x} \mid \mathbf{s}) .
$$

Equation 4 says that the interferometric gradient (or migration image) is formed by smearing the weighted crosscorrelogram residual along the associated migration ellipses. In the next subsection, we interpret the physical meaning of the gradient using the asymptotic Green's functions.
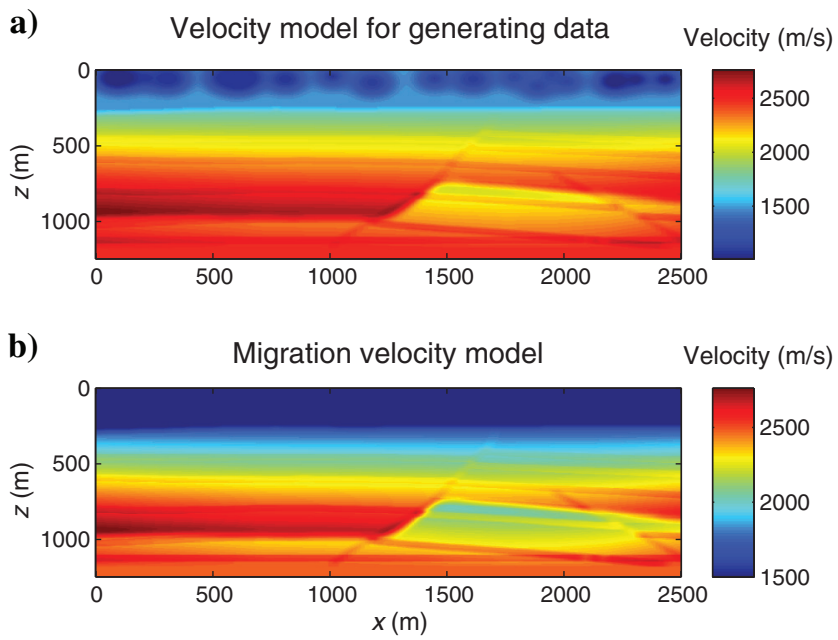

Figure 2. Velocity model used for (a) generating the observed data and (b) ILSM and LSM. 


\section{Interpretation of the ILSM gradient}

The asymptotic high-frequency Green's function for a reflection from a point $\mathbf{x}_{\mathbf{0}}$ in the subsurface is defined as

$$
D(\mathbf{g} \mid \mathbf{s})=A\left(\mathbf{g}, \mathbf{x}_{\mathbf{0}}, \mathbf{s}\right) e^{i \omega\left(\tau_{\mathbf{s x}_{\mathbf{0}}}+\tau_{\mathbf{x}_{\mathbf{0}} \mathbf{g}}\right)},
$$

where $\tau_{\mathbf{s x}_{0}}+\tau_{\mathbf{x}_{0} \mathbf{g}}$ is the reflection traveltime from the source $\mathbf{s}$ to the receiver $\mathbf{g}$ for a reflection at point $\mathbf{x}_{\mathbf{0}}$ in the subsurface and $A\left(\mathbf{g}, x_{0}, s\right)$ is an amplitude term that accounts for geometric spreading and the reflection coefficient. Similarly, the Green's function for the reference reflection shown in Figure 1 is defined as

$$
D(\mathbf{g} \mid \mathbf{s})_{\text {ref }}=A^{\prime}\left(\mathbf{g}, \mathbf{x}_{\text {ref }}, \mathbf{s}\right) e^{i \omega\left(\tau_{\mathbf{s x}_{\text {ref }}}+\tau_{\mathbf{x}_{\text {ref }} \mathbf{g}}\right)},
$$

where $\tau_{\mathbf{S x}_{\text {ref }}}+\tau_{\mathbf{x}_{\text {ref }} \mathbf{g}}$ is the calculated reflection time to the reference layer for a source at $\mathbf{s}$ and geophone at $\mathbf{g}$.

The predicted crosscorrelogram $\Phi(\mathbf{g} \mid \mathbf{s})$ can be obtained by crosscorrelating the predicted data with the picked reference reflection in

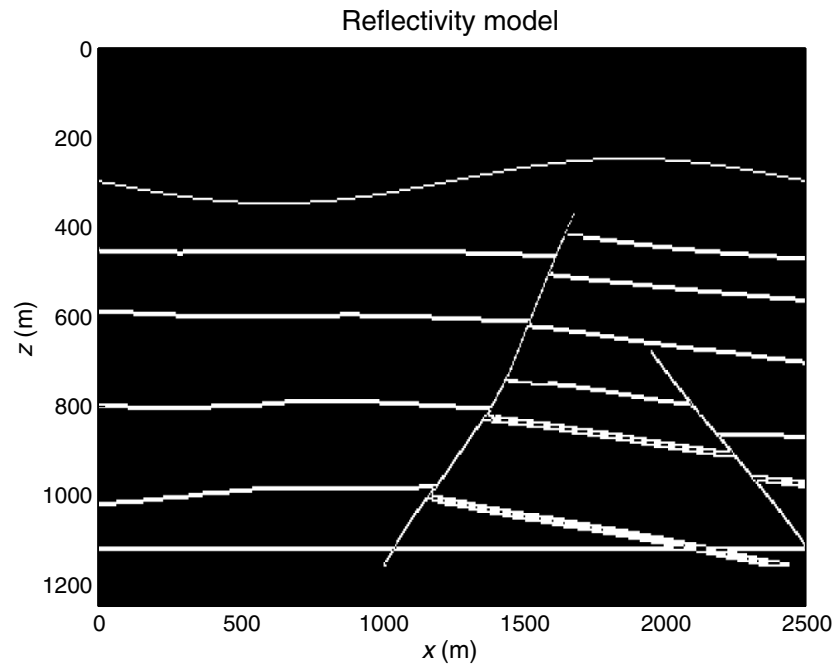

Figure 3. Reflectivity model used for generating data, in which the curved reflector on the top is chosen as the reference layer.
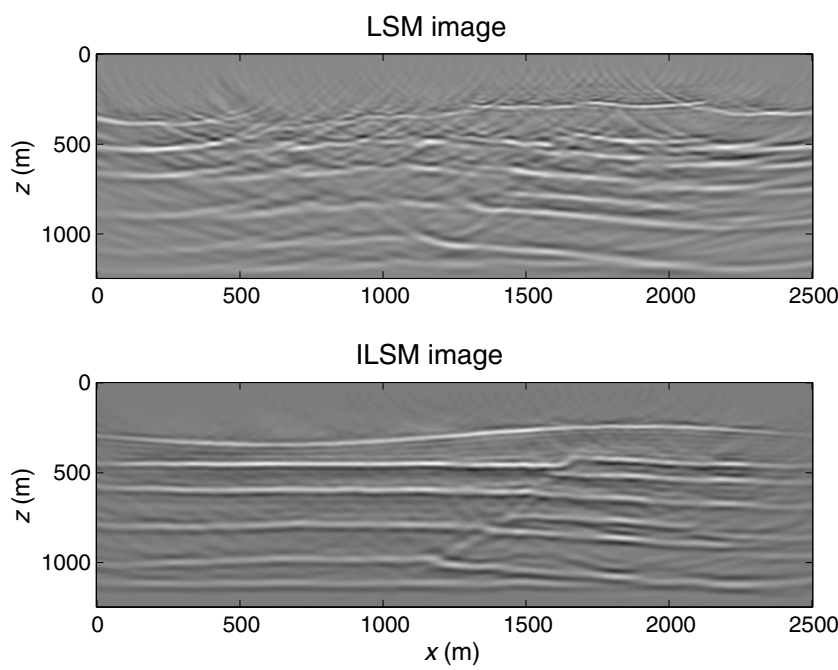

Figure 4. Migration images from (a) standard LSM and (b) ILSM. the predicted data. In the frequency domain, this predicted crosscorrelogram can be approximated as

$$
\Phi(\mathbf{g} \mid \mathbf{s})=D(\mathbf{g} \mid \mathbf{s}) e^{-i \omega\left(\tau_{\mathbf{s x}_{\mathbf{r e f}}}+\tau_{\mathbf{x}_{\text {ref }} \mathbf{g}}\right)},
$$

and the observed crosscorrelogram $\tilde{\Phi}(\mathbf{g} \mid \mathbf{s})$ can be calculated as

$$
\tilde{\Phi}(\mathbf{g} \mid \mathbf{s})=\tilde{D}(\mathbf{g} \mid \mathbf{s}) e^{-i \omega\left(\tilde{\tau}_{\mathbf{s x}_{\mathbf{r e f}}}+\tilde{\tau}_{\mathbf{x}_{\text {ref }} \mathbf{g}}\right)},
$$

where $\tilde{\tau}_{\mathbf{s x}_{\text {ref }}}+\tilde{\tau}_{\mathbf{x}_{\text {ref }} \mathbf{g}}$ is the picked reflection traveltime from the recorded data for a reflection corresponding to the reference layer for a source at $\mathbf{s}$ and a geophone at $\mathbf{g}$. Ignoring the normalization factor, the weighted residual $\Delta \Phi(\mathbf{g} \mid \mathbf{s})$ in equation 4 can be calculated as

$$
\Delta \Phi(\mathbf{g} \mid \mathbf{s})=w(\mathbf{g} \mid \mathbf{s}) \Phi(\mathbf{g} \mid \mathbf{s})-\tilde{\Phi}(\mathbf{g} \mid \mathbf{s})
$$

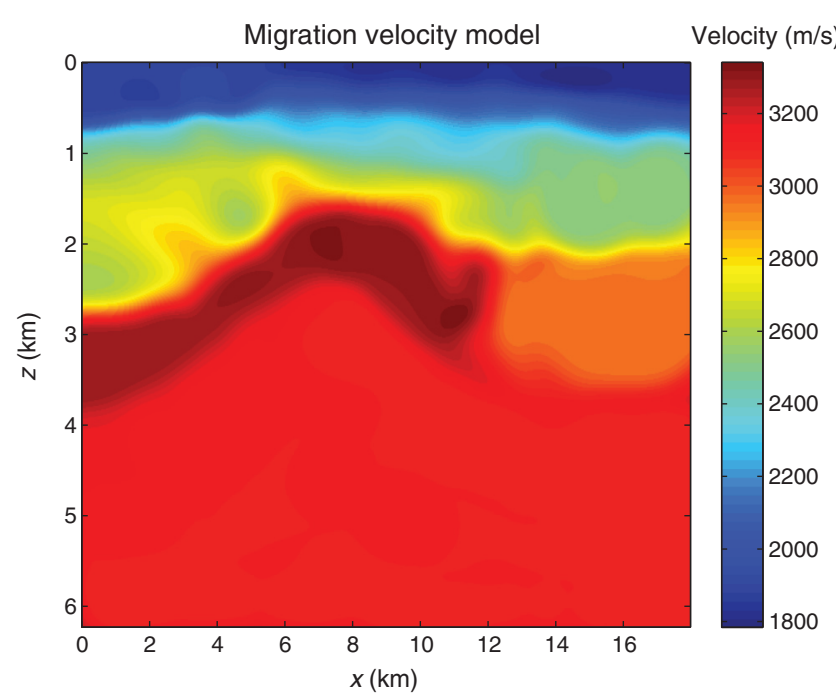

Figure 5. Migration velocity model.

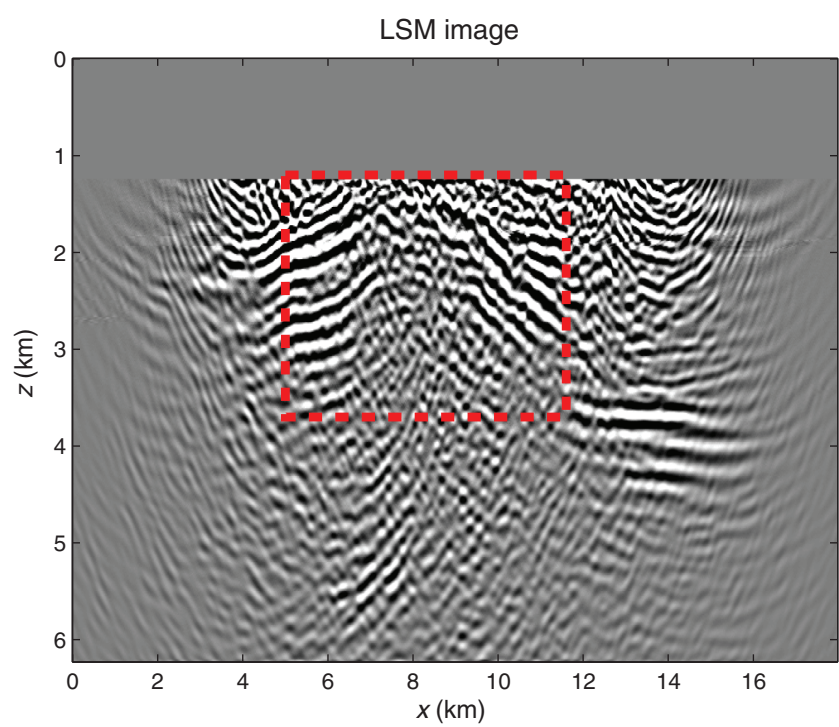

Figure 6. Standard LSM image obtained using the velocity model shown in Figure 5. 
Substituting the residual $\Delta \Phi(\mathbf{g} \mid \mathbf{s})$ and $D(\mathbf{g} \mid \mathbf{s})_{\text {ref }}$ from equation 7 into equation 4 we get the following expression for the gradient:

$$
\frac{\partial \epsilon}{\partial s(\mathbf{x})}=\sum_{\omega} \sum_{\mathbf{s}} \sum_{\mathbf{g}} \Delta \Phi(\mathbf{g} \mid \mathbf{s}) e^{-i \omega\left(\tau_{\mathbf{s x}}+\tau_{\mathbf{x g}}-\tau_{\mathbf{s}} \mathbf{r e f}-\tau_{\mathbf{x}_{\mathbf{r e f}} \mathrm{g}}\right)}
$$

where the amplitude term associated with $D(\mathbf{g} \mid \mathbf{s})_{\text {ref }}$ is conveniently ignored. Substituting equations 8 and 9 in equation 10 we get the following equation:

$$
\Delta \Phi(\mathbf{g} \mid \mathbf{s})=w(\mathbf{g} \mid \mathbf{s}) D(\mathbf{g} \mid \mathbf{s}) e^{-i \omega \tau_{\mathbf{s g}}^{\mathrm{ref}}}-\tilde{D}(\mathbf{g} \mid \mathbf{s}) e^{-i \omega \tilde{\tau}_{\mathbf{s g}}^{\mathrm{ref}}},
$$
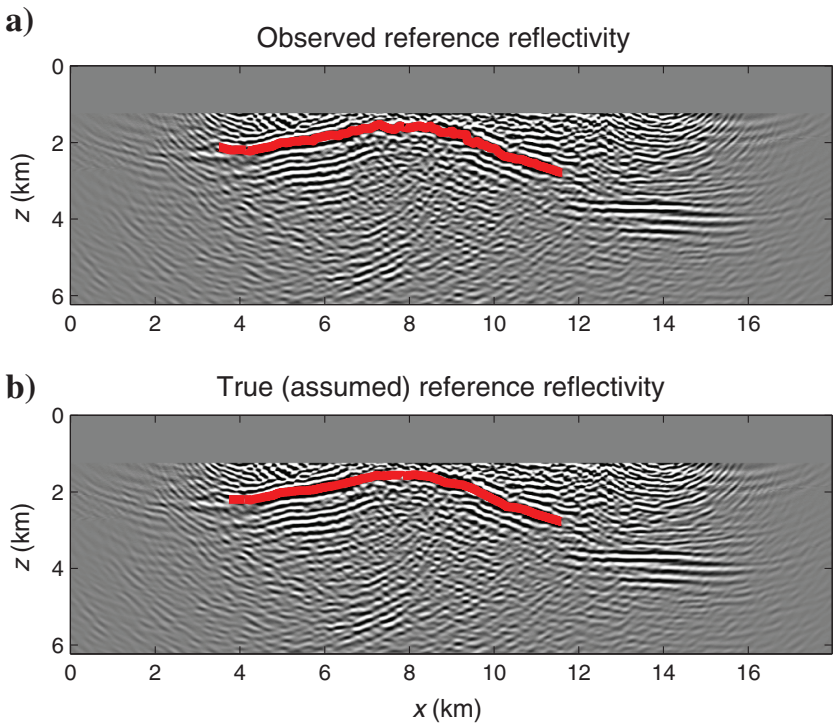

Figure 7. (a) Observed reference interface (shown in red) picked from the LSM image. (b) Calculated reference (shown in red) is a smoothed-out version of the observed reference interface.

a)

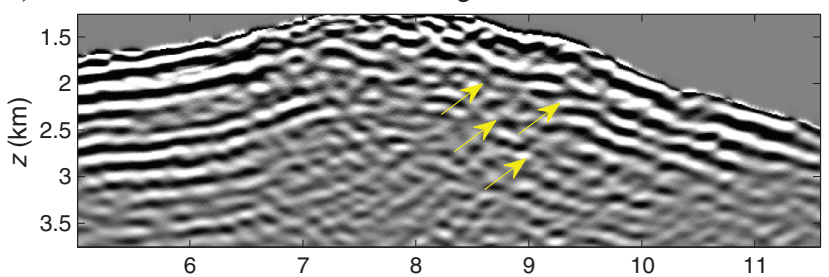

b)

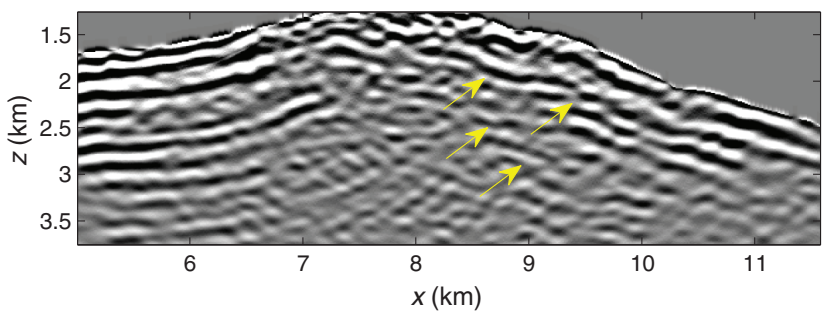

Figure 8. Magnified views of the red dashed box in Figure 6 . (a) Standard LSM image and (b) ILSM image. where $\tau_{\mathbf{s g}}^{\text {ref }}=\tau_{\mathbf{s x}_{\text {ref }}}+\tau_{\mathbf{x}_{\text {ref }} \mathbf{g}}$ is the predicted traveltime for the reference reflection and $\tilde{\tau}_{\mathbf{s g}}^{\text {ref }}=\tilde{\tau}_{\mathbf{s x}_{\text {ref }}}+\tilde{\tau}_{\mathbf{x}_{\text {ref }} \mathbf{g}}$ is the traveltime for the reference reflection in the observed data. Using the expression for $\Delta \Phi(\mathbf{g} \mid \mathbf{s})$ in equation 12 , equation 11 simplifies to

a)
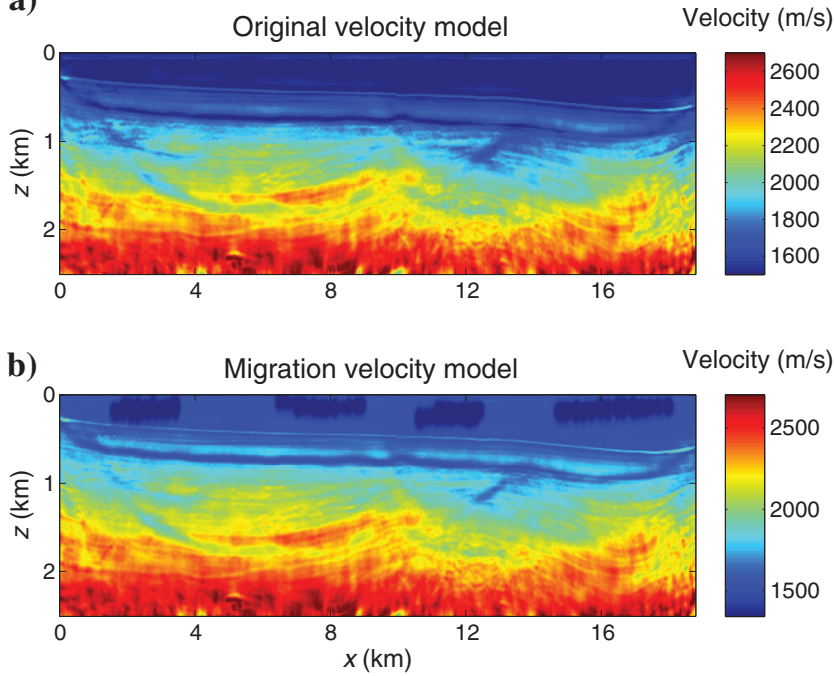

Figure 9. (a) Original velocity model and (b) the migration velocity model. Low-velocity anomalies are added in the shallow part of the migration velocity model. The sea bottom is chosen as the reference reflector.

a)
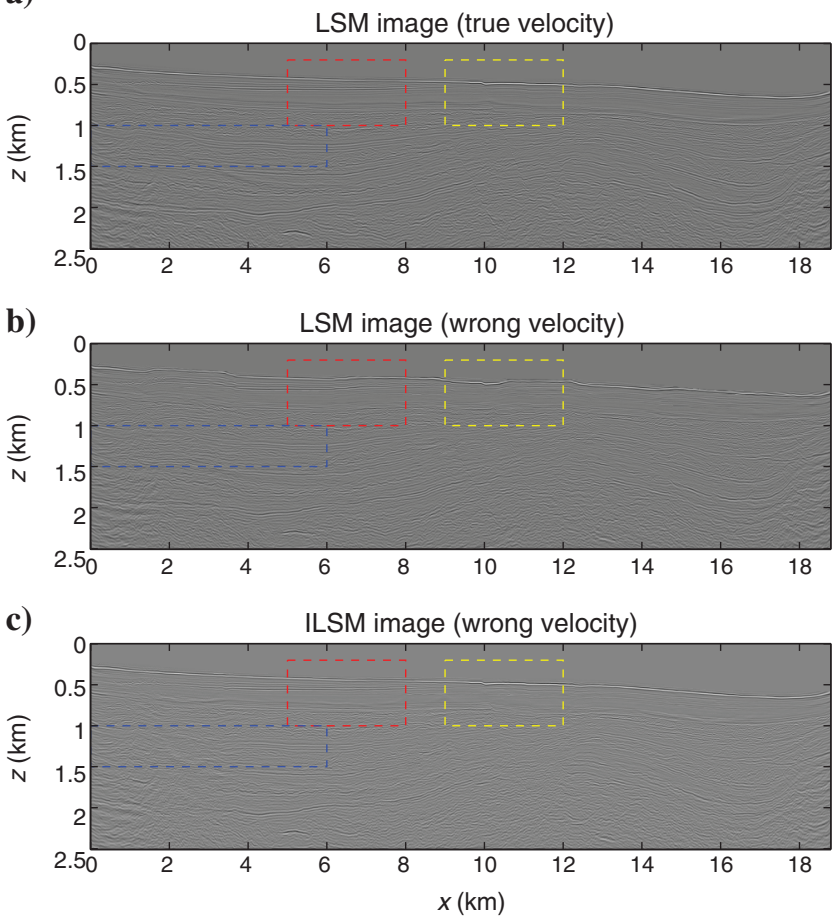

Figure 10. (a) LSM image from the true velocity model; the sea bottom is chosen as the reference reflector, (b) LSM image with an erroneous velocity model, and (c) ILSM image using an erroneous velocity model. 
a) LSM image (true velocity)

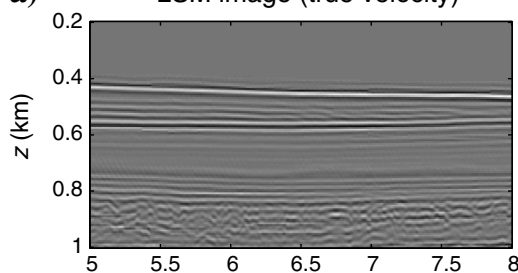

b)

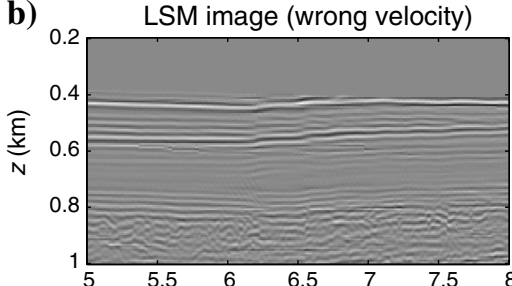

c) ILSM image (wrong velocity)

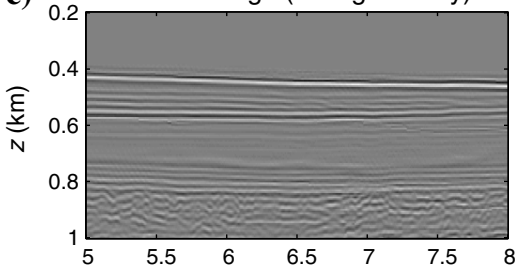

d)

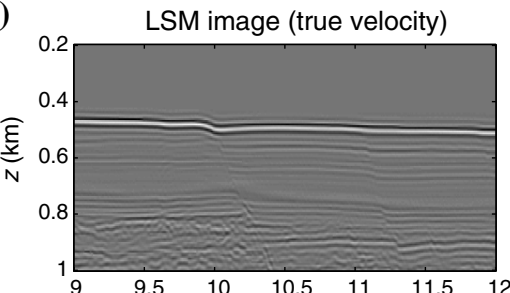

e)

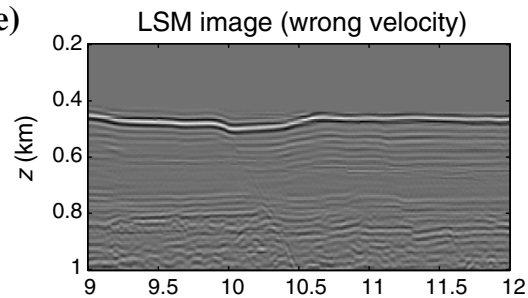

f)

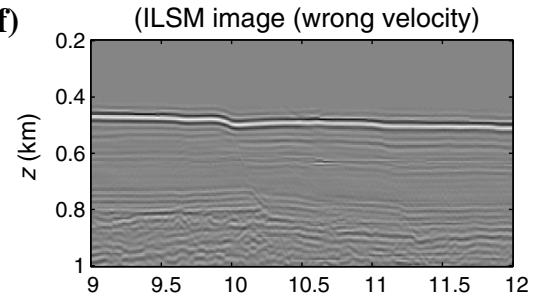

Figure 11. Magnified views of red and yellow dashed boxes in Figure 10. (a) LSM image (red box) using the true velocity model, (b) LSM image (red box) using an erroneous velocity model, (c) ILSM image (red box) using an erroneous velocity model, (d) LSM image (yellow box) using the true velocity model, (e) LSM image (yellow box) using an erroneous velocity model, and (f) ILSM image (yellow box) using an erroneous velocity model. a)

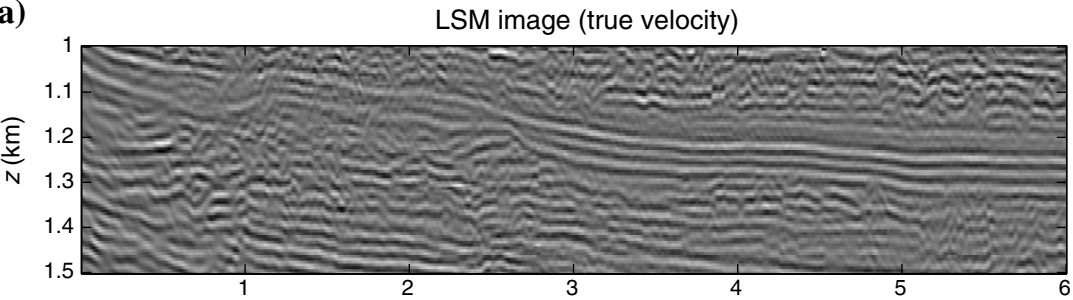

b)

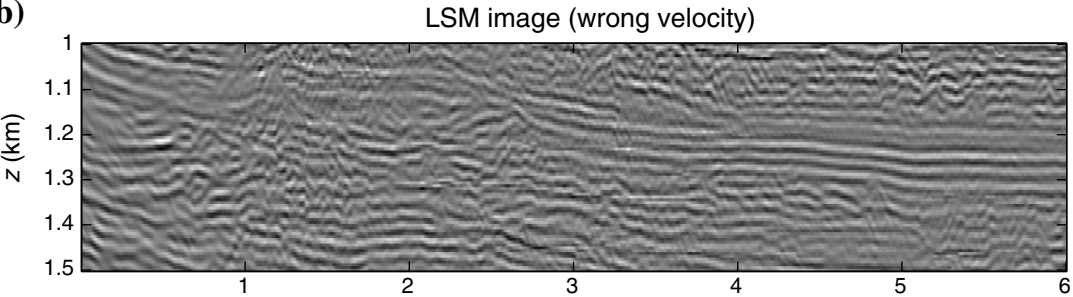

c)

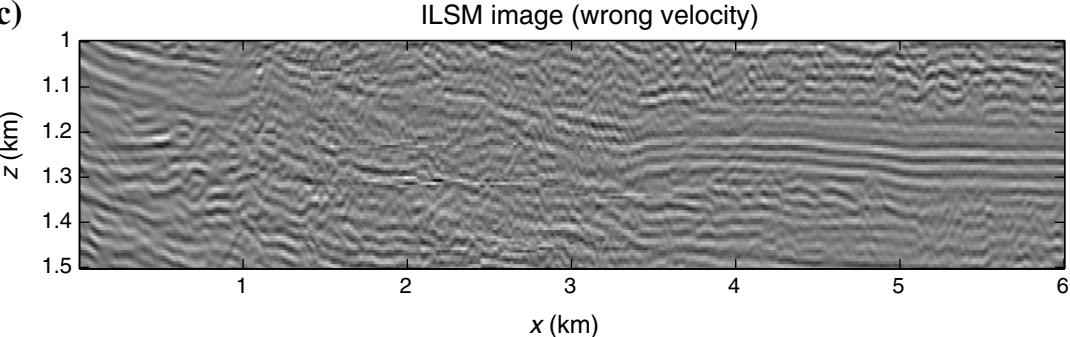

Figure 12. Magnified views of blue dashed box in Figure 10. (a) LSM image from the true velocity model, the sea bottom is chosen as the reference reflector, (b) LSM image with an erroneous velocity model, and (c) ILSM image with an erroneous velocity model. 

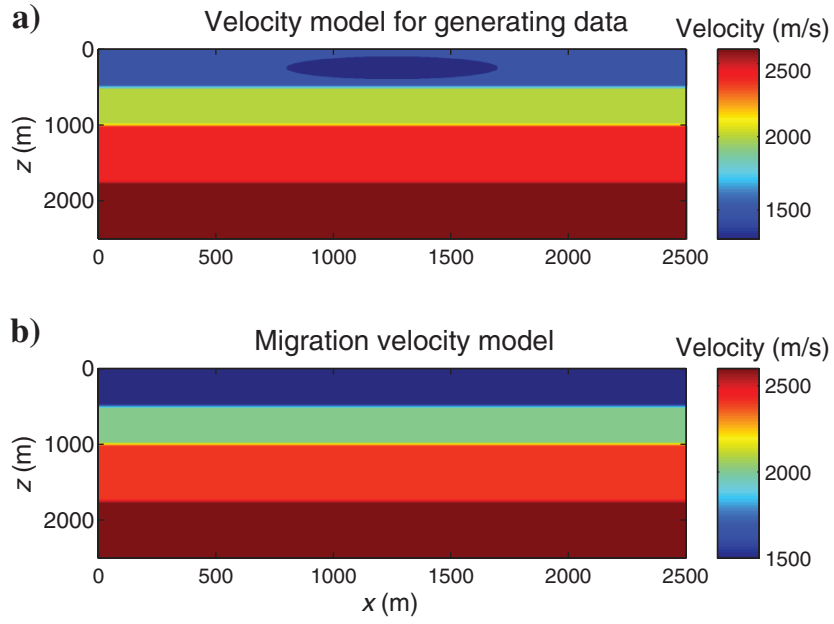

Figure 13. (a) Velocity model used for generating the observed data and (b) migration velocity without the low-velocity anomaly.

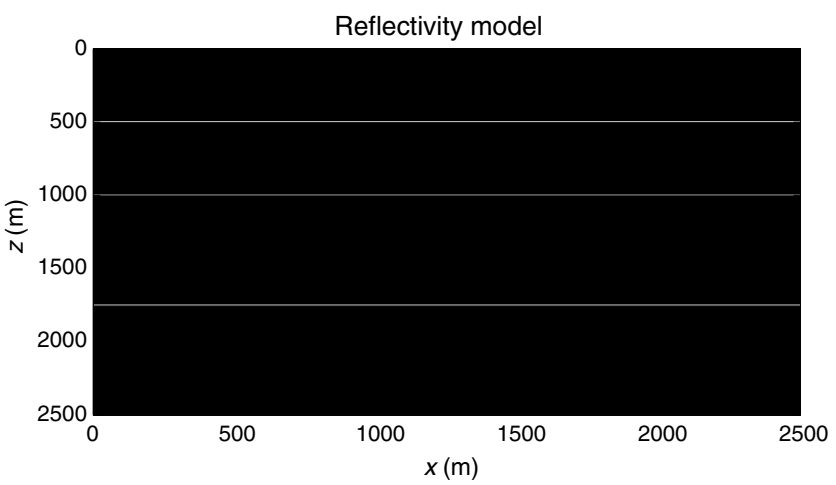

Figure 14. Reflectivity model used for generating data. The reflector on the top is chosen as the reference layer.

a)

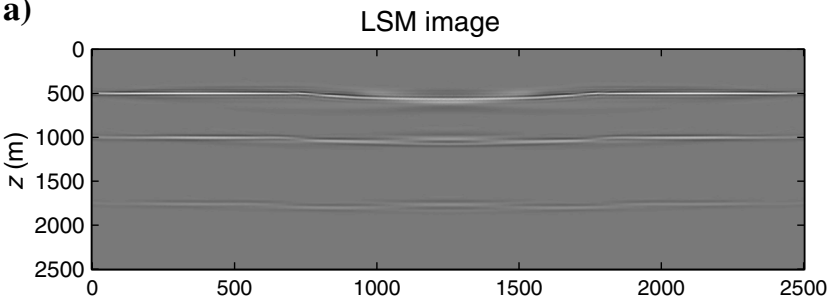

b)

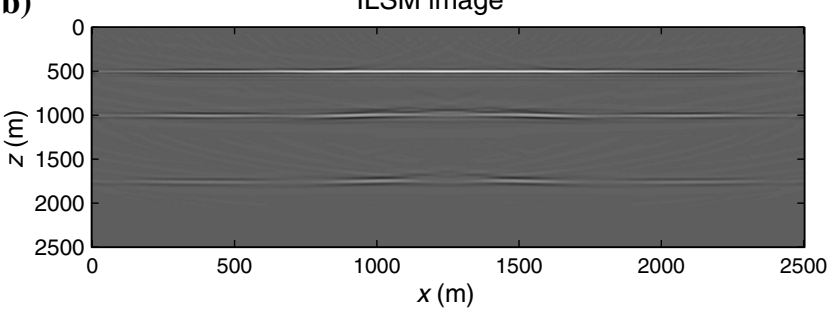

Figure 15. (a) LSM image and (b) ILSM image obtained using the migration velocity model shown in Figure $13 \mathrm{~b}$.

$$
\begin{aligned}
& \frac{\partial \epsilon}{\partial s(\mathbf{x})}=\sum_{\omega} \sum_{\mathbf{s}} \sum_{\mathbf{g}} \\
& \times\left[w(\mathbf{g} \mid \mathbf{s}) D(\mathbf{g} \mid \mathbf{s})-\tilde{D}(\mathbf{g} \mid \mathbf{s}) e^{i \omega(\overbrace{\mathbf{s g}}^{\text {ref }}-\tilde{\tau}_{\mathbf{s g}}^{\text {ref }})}\right] \overbrace{e^{-i \omega\left(\tau_{\mathbf{s x}}+\tau_{\mathbf{x g}}\right)}}^{\text {migration kernel }}
\end{aligned}
$$

The term $\delta^{\text {ref }}$ is the timing error of the reference reflector due to statics because the recorded data contain statics but the predicted does not; the product of this exponential with the recorded data $\tilde{D}(\mathbf{g} \mid \mathbf{s})$ eliminates this statics term. At each step of ILSM, the gradient is calculated by migrating the weighted crosscorrelogram residual. The residual is calculated by temporally shifting the observed trace by $\delta^{\text {ref }}$ and then subtracting it from the weighted predicted trace $w(\mathbf{g} \mid \mathbf{s}) D(\mathbf{g} \mid \mathbf{s})$. Temporally shifting the observed trace by $\delta^{\text {ref }}$ is similar to applying a static correction to the data. The next subsection describes the workflow of the method.

\section{Workflow}

- Define a reference reflector in the reflectivity model and window the corresponding reference reflection in the observed data.

- Crosscorrelate the observed data with observed reference reflection data to get the observed crosscorrelogram. Now calculate the interferometric residual in equation 4 . Note, there is no explicit picking or statics calculation in each trace.

- Calculate the gradient $\mathbf{g}^{k+1}$ in equation 4 and update the search direction $\mathbf{d}^{k}$ using the conjugate gradient method (Nocedal and Wright, 2006)

$$
\mathbf{d}^{k+1}=-\mathbf{g}^{k+1}+\beta \mathbf{d}^{k}
$$

where $\beta$ is calculated using the Fletcher-Reeves formula

$$
\beta=\frac{\left(\mathbf{g}^{k+1}, \mathbf{g}^{k+1}\right)}{\left(\mathbf{g}^{k}, \mathbf{g}^{k}\right)}
$$

- Compute the step length $\alpha$ by a line-search method.

- Update the migration image $\mathbf{m}^{k+1}$ by

$$
\mathbf{m}^{k+1}=\mathbf{m}^{k}+\alpha \mathbf{d}^{k+1} .
$$

- Calculate the new predicted crosscorrelogram using equation 1 .

The effectiveness of ILSM in mitigating statics at the near surface is now illustrated with numerical examples.

\section{NUMERICAL RESULTS}

ILSM is tested on a 2D fault model derived from the 3D SEGEAGE salt model, except several low-velocity anomalies are introduced in the near-surface region. Figure 2 depicts the true velocity model for generating the observed data and the migration velocity model. The reflectivity model for generating the data is shown in Figure 3, and the curved reflector on the top is chosen as the reference reflector. Kirchhoff-Born modeling is used to generate the 
synthetic data. A fixed-spread acquisition is used with 50 shots sampled every $50 \mathrm{~m}$ on the surface. Each shot is recorded with 501 receivers spaced every $5 \mathrm{~m}$ on the surface with a recording time of $4 \mathrm{~s}$. Kirchhoff migration is used for LSM and ILSM in imaging these data. The conventional LSM and ILSM images obtained after 20 iterations are shown in Figure 4, in which the reflectors in the ILSM image are better focused than the LSM image. The deeper reflectors are moderately distorted because the timing error of the reference reflection does not exactly account for statics corrections in the later reflections. But the defocusing due to the static errors is reduced, and a better migration image is inverted. As shown later, the image distortion at deeper depths can be remedied by selecting a deeper reference interface.

ILSM is also tested on a 2D ocean-bottom seismic (OBS) data set from the Gulf of Mexico (GoM). There are 26 OBS receivers spread out at an interval of $400 \mathrm{~m}$ at a depth of $45 \mathrm{~m}$, and 360 sources are placed on the sea surface with a shot interval of $50 \mathrm{~m}$. Each trace is $12 \mathrm{~s}$ long. Figure 5 shows the migration velocity model. The standard LSM image is shown in Figure 6. The picked red reference interface in Figure 7a is defined as the observed reference reflector. Because we do not know the true location and the shape of the layer, a smoothly varying reference interface shown in Figure $7 \mathrm{~b}$ is chosen as the true reference reflector. By Born modeling these two reflectivity models, we can evaluate the observed and calculated reference reflection traces. The magnified views shown in Figure $8 \mathrm{a}$ and $8 \mathrm{~b}$ indicate the ILSM image in the red dashed box in Figure 6. The yellow arrows indicate the regions that show improvement.

ILSM is also tested on another GoM data set. The data consist of 515 shot gathers with a shot interval of $37.5 \mathrm{~m}$. There are 480 receivers in each shot gather with receivers placed every $12.5 \mathrm{~m}$ along a line. To replicate a situation in which data contain statics, low-velocity errors are incorporated into the original tomogram. The original tomogram is shown in Figure 9a, and the LSM image obtained after 10 iterations using this velocity model is shown in Figure 10a. The sea-bottom reflector is chosen as the reference reflector for ILSM. Errors are added to the original tomogram, and the resulting erroneous velocity model is shown in Figure 5b. The LSM and ILSM images obtained after 10 iterations using this velocity model are shown in Figure 10b and 10c, respectively. The magnified views of the dashed red and yellow boxes in Figure 10 are

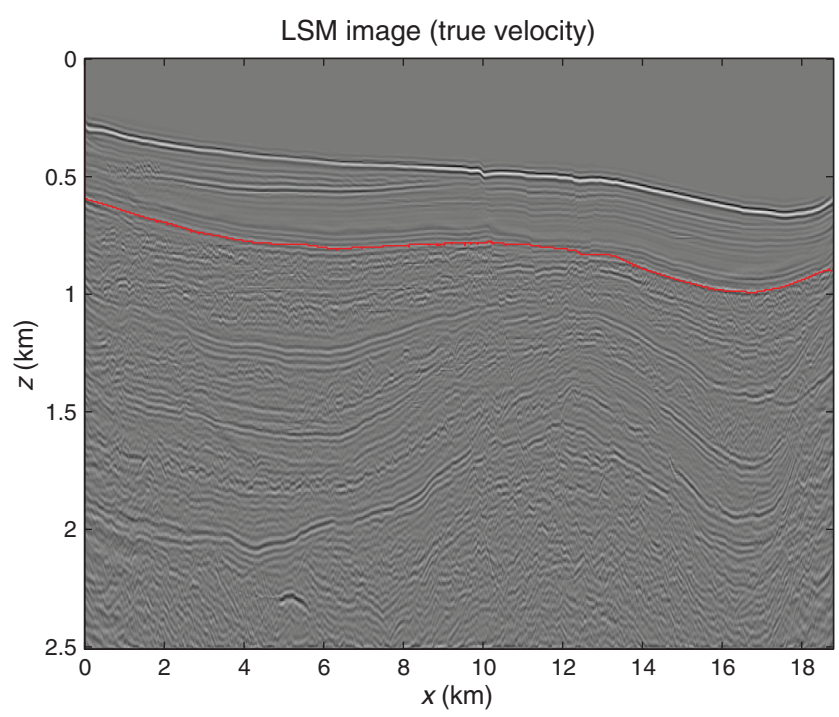

Figure 17. Migration image showing the reference reflector marked in red.
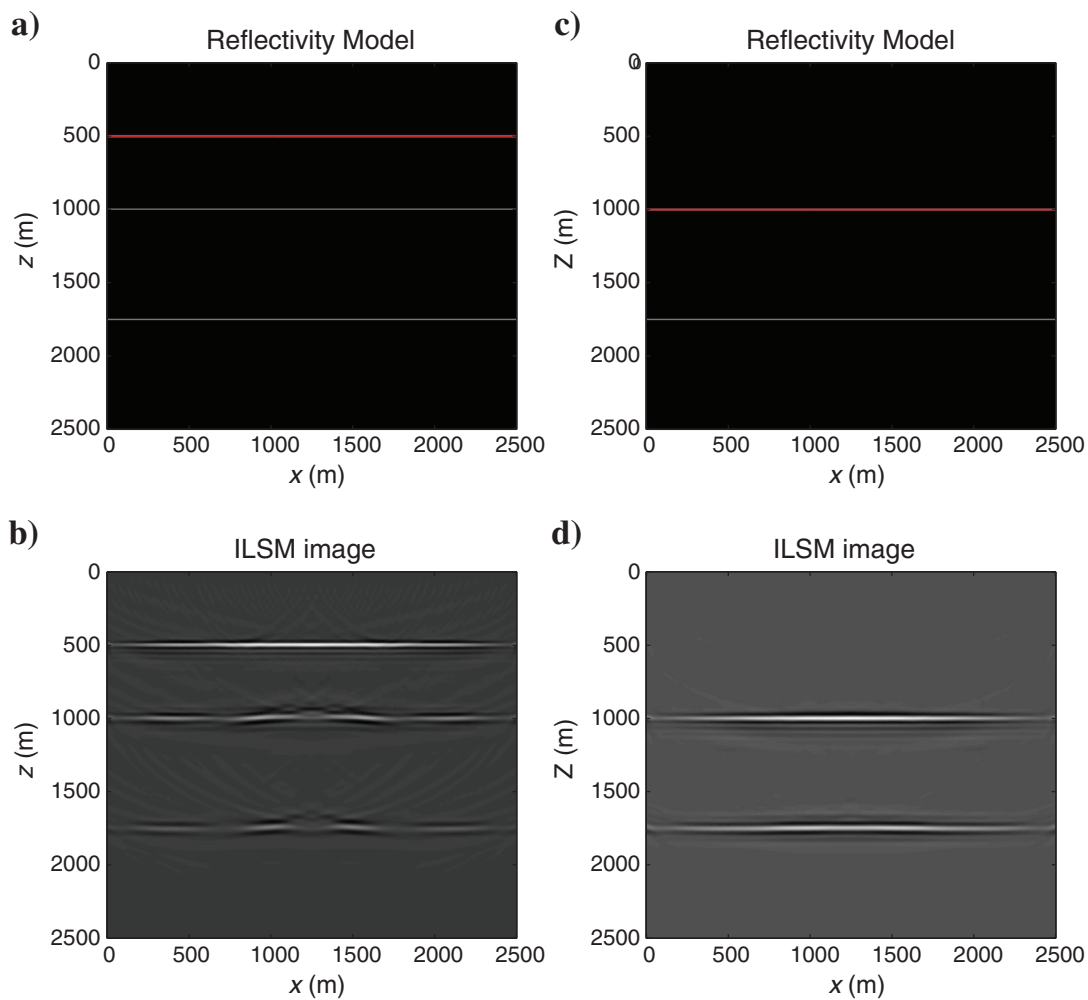

Figure 16. (a) Reflectivity image used for generating data; the top-most reflector (indicated in red) is chosen as the reference reflector for ILSM, and the corresponding ILSM image is shown in panel (b). (c) The reflectivity image in which the reflector in the middle is chosen as the reference reflector, and the corresponding ILSM image is shown in panel (d). 
Figure 18. (a) LSM image from the true velocity model; the deeper reflector shown in Figure 17 is chosen as the reference reflector. (b) LSM image with an erroneous velocity model. (c) ILSM image using an erroneous velocity model.

Figure 19. Magnified views of the blue dashed box in Figure 18. (a) LSM image from the true velocity model, (b) LSM image with an erroneous velocity model, and (c) ILSM image using an erroneous velocity model (the reference reflector is shown in Figure 17).
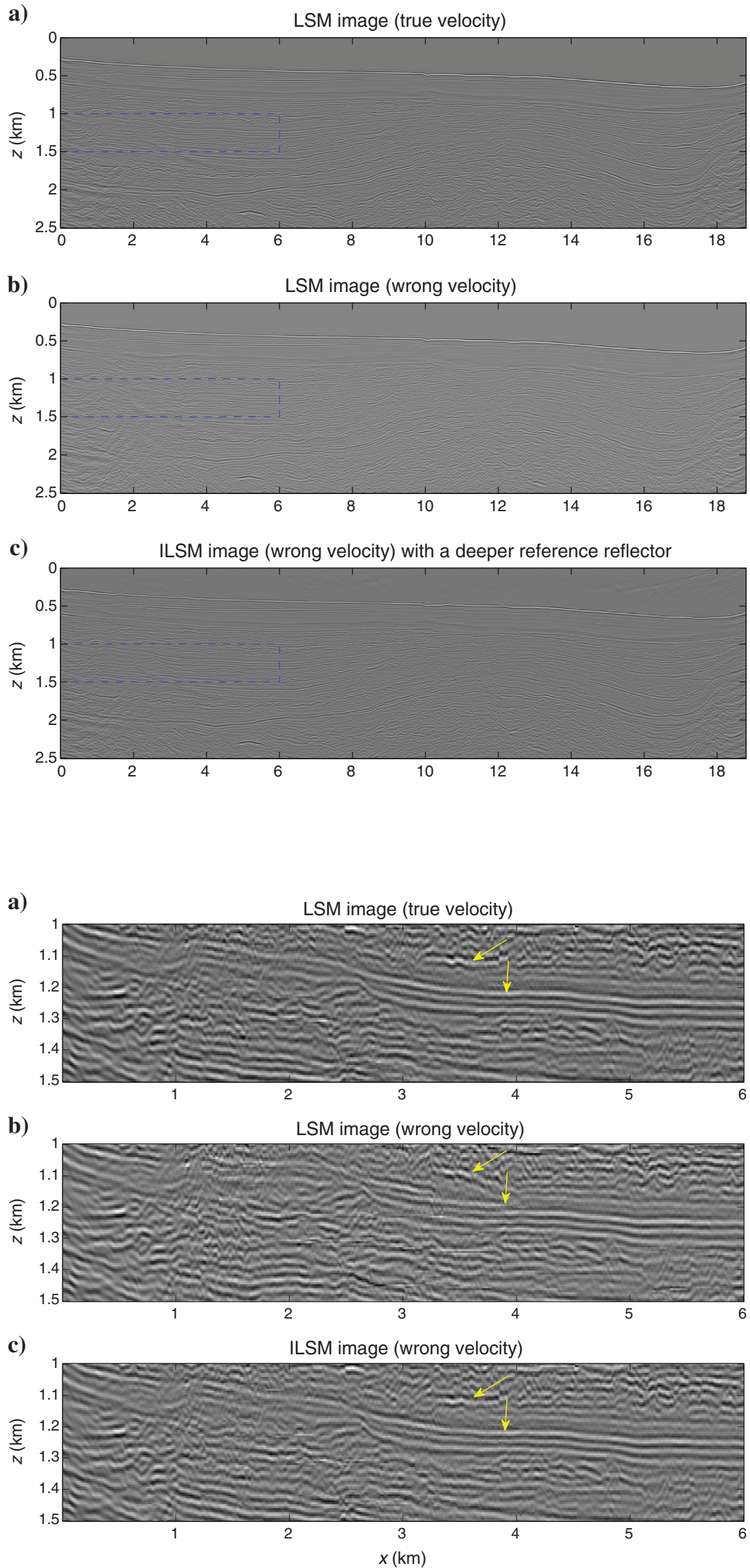
shown in Figure 11. The LSM image is defocused because of the velocity model errors; in contrast, ILSM is able to accurately image the shallow reflectors because they are close to the reference seabottom reflector. However, the deeper reflectors located away from the sea-bottom reflector are not imaged accurately. This can be seen in Figure 12 that shows the magnified views of the blue dashed boxes in Figure 10. To image the deeper parts of the subsurface, we can iteratively move the reference reflector to deeper levels and use it to accurately image even deeper reflectors. This stair-stepping strategy is now applied to synthetic and field data.
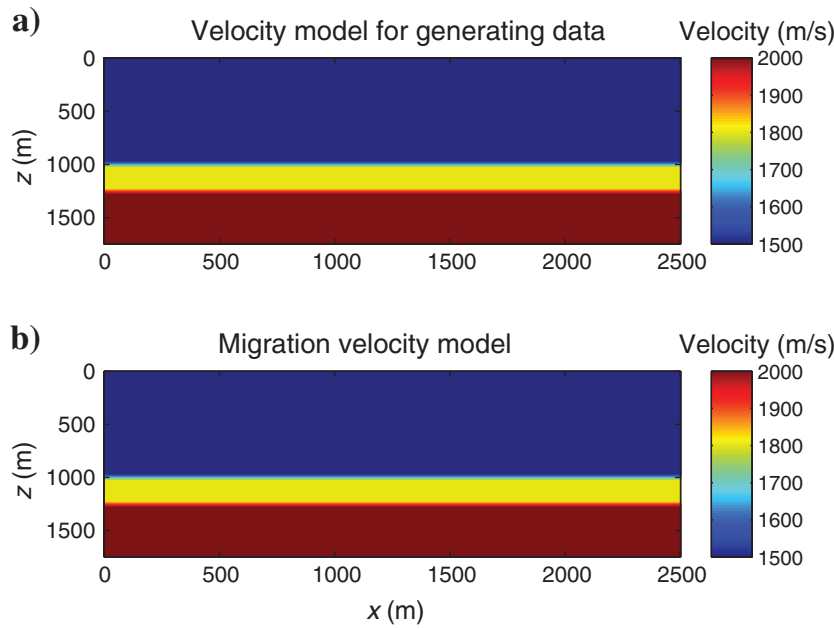

Figure 20. (a) Velocity model used for generating the baseline survey and (b) migration velocity model for the baseline survey.
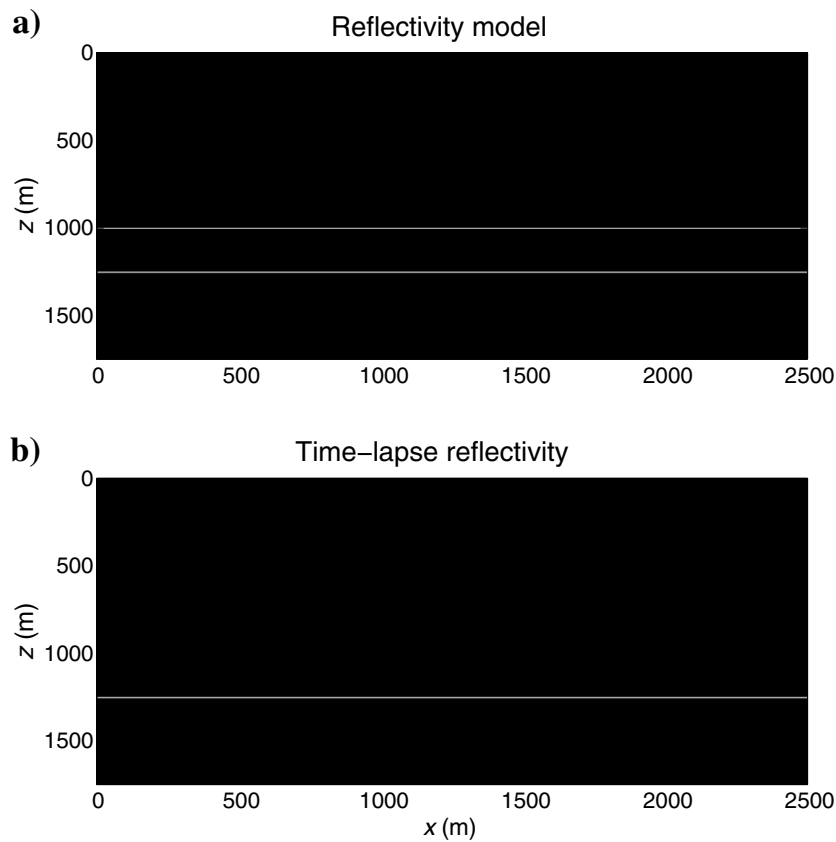

Figure 21. (a) Reflectivity model used for generating the data for the baseline survey. (b) Time-lapse reflectivity, the difference between monitor and base survey reflectivity. The sea bottom remains the same; hence, it gets canceled out.

\section{Stair-stepping approach}

Synthetic acoustic data are generated for the four-layer model in Figure 13a, and the migration velocity model is shown in Figure 13b. The reflectivity model used for generating the data is shown in Figure 14, in which the reflector on the top is chosen as the reference reflector for ILSM. A fixed spread acquisition with 50 shot gathers recorded by 501 receivers is used. The shot and receiver intervals are 50 and $5 \mathrm{~m}$, respectively. The LSM and ILSM images are shown in Figure 15; the bottom-most reflector is best imaged by ILSM, although it is still distorted. Now we select the second reflector from the top as the reference reflector (marked in red) as shown in Figure 16c. The ILSM images obtained by selecting the first and the second reflectors as reference reflectors are shown in Figure 16b and 16d, respectively. We can see the deepest reflector is better delineated when we selected a deeper reflector as a reference reflector, because the closer a reflector is to the reference interface the more accurately it is imaged.

The stair-stepping strategy is now applied to the GoM data. The reference reflector (marked in red) is now lowered to a deeper level as shown in Figure 17. Figure 18 shows the migration images computed by LSM with the true and wrong velocity models and the ILSM image with the deeper reflector as a reference reflector. Magnified views of the blue dashed boxes in Figure 18 are shown in Figure 19 and demonstrate that the deeper reference reflector for ILSM provides a more reliable image near that depth.

\section{Time-lapse imaging}

A significant application of ILSM is for time-lapse studies in which the environment can significantly change from one survey to the next. For example, an early marine survey might have rough sea surface conditions that introduce statics to the recorded reflections compared with a later survey with a calm sea surface. These 4D statics can be removed with ILSM. Moreover, selecting a stationary reference interface above the reservoir will effectively allow both surveys to be conducted just above the reservoir and not be prone to the changes in the acquisition.
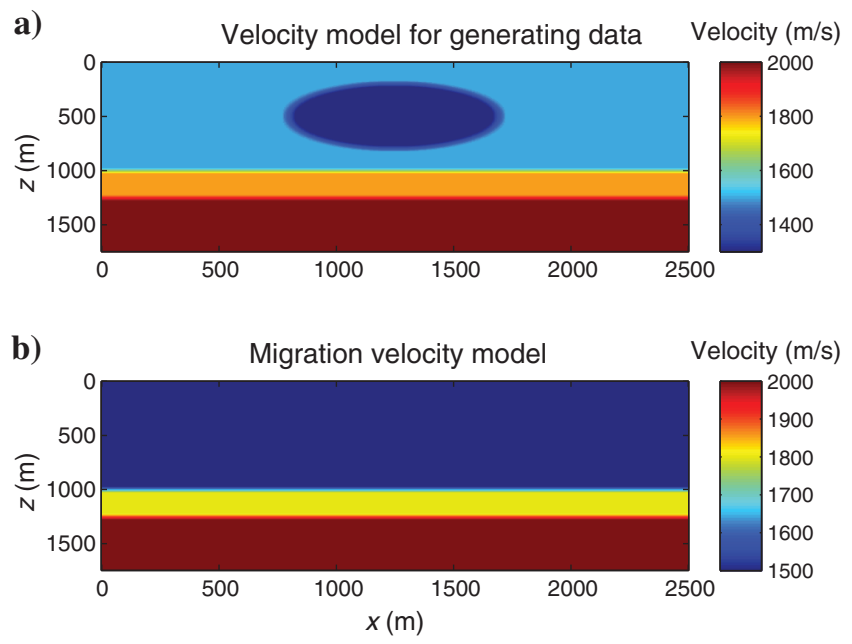

Figure 22. (a) Velocity model used for generating the observed data for the monitor survey and (b) migration velocity model for the monitor survey. 
To test this idea, a fixed-spread acquisition geometry is used for the baseline and the monitor surveys. The velocity model used for generating the data and imaging is the same for the baseline survey shown in Figure 20. The reflectivity model used for generating the data is shown in Figure 21a: The shallow reflector can be thought of as the sea bottom, and the second reflector is taken to be the top interface of a reservoir. The velocity model used for generating the monitor data is shown in Figure 22a. It contains a low-velocity anomaly in the water layer which generates static shifts in the data and hampers the repeatability of the time-lapse survey. The migration velocity model for the monitor survey is shown in Figure 22b. The net change in the reflectivity over the course of the two surveys is shown in Figure 21b, in which only reservoir reflectivity has changed and the sea bottom remains the same. In addition, the source and receiver positions of the monitor survey vary randomly and introduce random static shifts in the data. These statics can be thought of as the effect of varying tidal conditions from one survey to another.

LSM and ILSM (the sea bottom is used as a reference reflector) are used to obtain the migration images for the baseline and monitor surveys, and the results are shown in Figures 23 and 24. The timelapse image obtained by ILSM shows reflectivity changes at the reservoir reflector and is immune to the time-lapse statics. In contrast, the LSM image mispositions the reservoir reflector because it is affected by the time-lapse statics.

\section{Sensitivity of ILSM to errors in selection of the reference reflector}

A fixed-spread acquisition is used with 50 shots placed at an interval of $40 \mathrm{~m}$. Each shot gather has 401 traces spaced at an interval of $5 \mathrm{~m}$. The velocity model used for generating the data is shown in
Figure 23. (a) LSM image for the baseline survey, (b) ILSM image for the baseline survey, (c) LSM image for the monitor survey, and (d) ILSM image for the monitor survey.
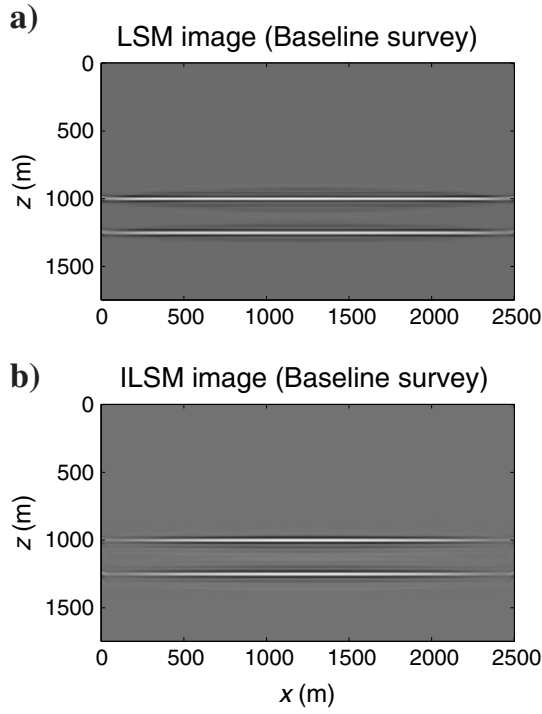
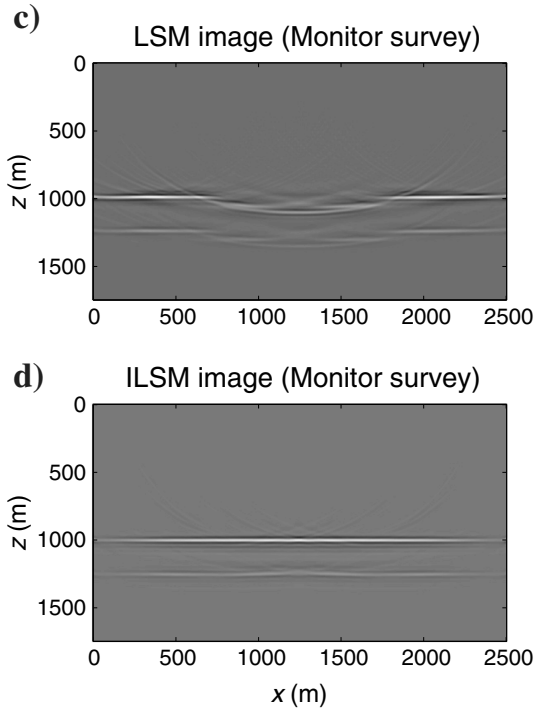

a)
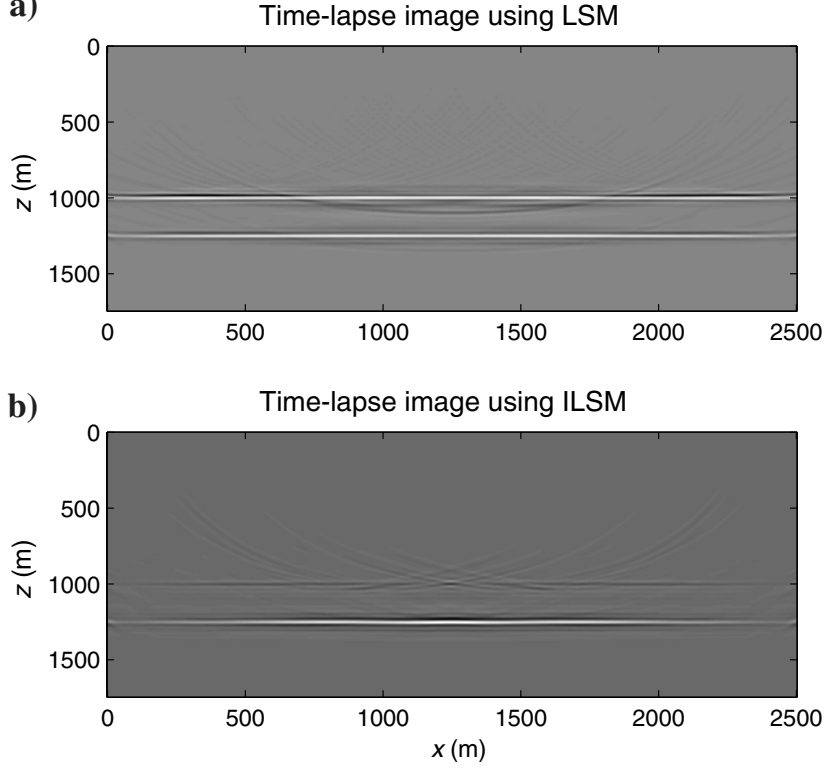

Figure 24. Time-lapse images using (a) LSM and (b) ILSM.
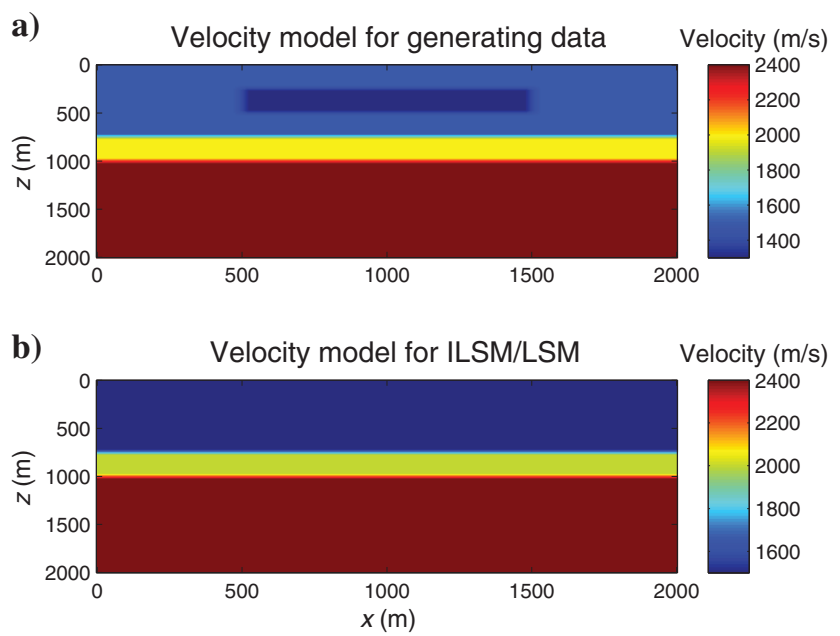

Figure 25. Velocity model used for (a) generating the observed data and (b) LSM/ILSM. 
Figure 25a, and the migration velocity model is shown in Figure 25b. One of the observed common shot gathers is shown in Figure 26a. The reflectivity model used to generate the data is shown in Figure 26b, and the reflector marked in yellow is chosen as the reference reflector. Migration velocity model along with the assumed reference reflector is used to calculate the reference reflection data shown in Figure 26c, using this as reference observed reference reflection data is windowed from the observed data. The window used for this example was two periods on the either side of the calculated reference reflection. The windowed reference reflection is shown in Figure 26d. The observed crosscorrelogram obtained by crosscorrelating the observed data, and observed reference reflection is shown in Figure 26e. According to equation 4, the crosscorrelogram is then convolved with the calculated reference reflection to obtain the residual which is to be backpropagated. This residual is shown in Figure 26f. The ILSM image after choosing the correct reference reflector is shown in Figure 27a, and the yellow dashed lines indicate the correct location of the reflectors in the reflectivity model shown in Figure 26b. The yellow lines coincide with the imaged reflectors. If a shallower reference reflector was incorrectly chosen as the reference reflector the target reflector is also imaged at a shallower depth in comparison with its original location. This is shown in Figure 27b, the reference reflector is chosen $75 \mathrm{~m}$ above the actual depth; here yellow lines show the correct locations of the reference and the target reflector. Similarly, if a deeper reference

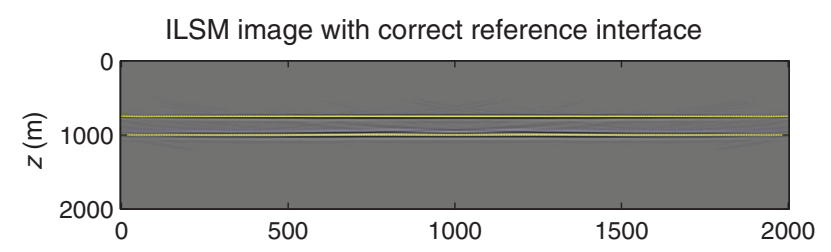

ILSM image with a shallower reference interface

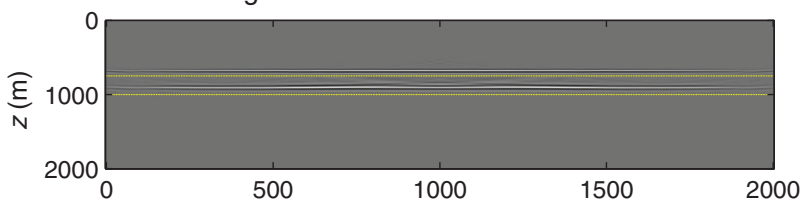

ILSM image with a deeper reference interface

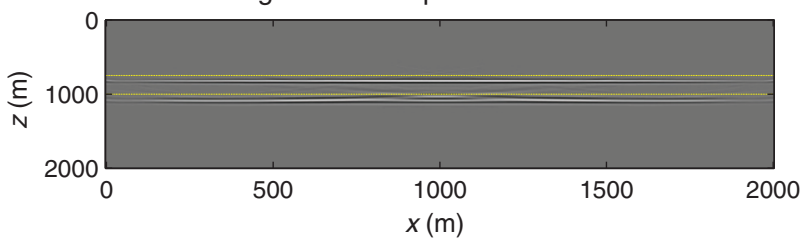

Figure 27. (a) ILSM image with the correct location of reference reflector, (b) ILSM image obtained using a shallower reference interface than the actual as reference reflector, and (c) ILSM image obtained using a deeper reference interface than the actual as reference reflector. a)

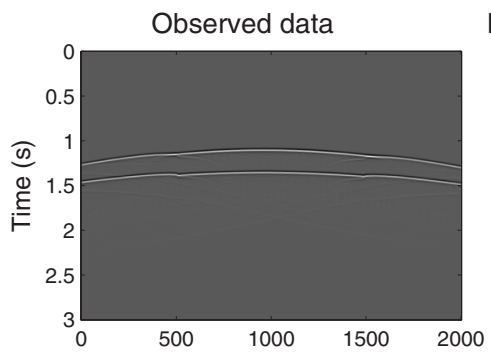

b)

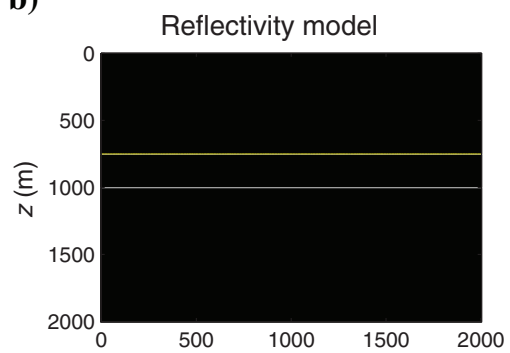

c)

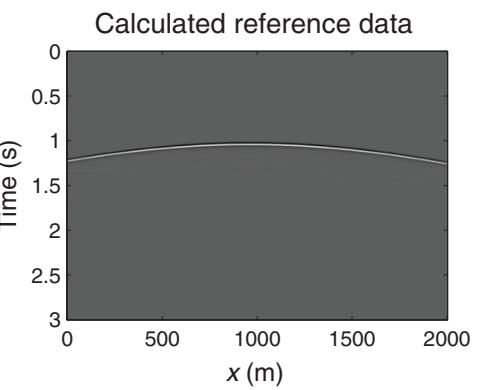

d)

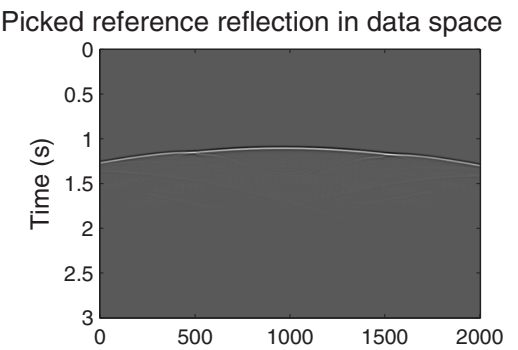

e)

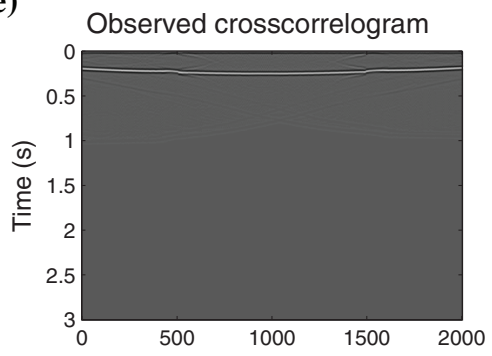

f)

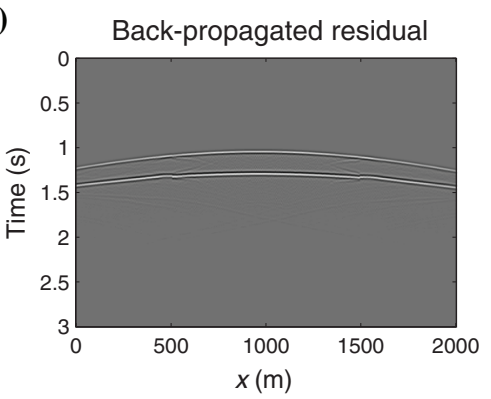

Figure 26. (a) Observed data generated from the velocity model shown in Figure 25a. (b) Reflectivity model used to generate the observed data; the reflector marked in yellow is used as the reference reflector. (c) Calculated reference reflector data generated using the reference interface and velocity model shown in Figure 25b. (d) Windowed observed reference reflection data. (e) Observed crosscorrelogram and (f) the back-propagated residual. 
interface is incorrectly chosen as reference reflector, the target reflector is also incorrectly imaged at a deeper depth. This is shown in Figure 27c, where the reference reflector is chosen $75 \mathrm{~m}$ below the actual depth; here yellow lines show the correct locations of the reference and the target reflector.

\section{CONCLUSIONS}

ILSM can mitigate the defocusing of migration images caused by source and receiver statics. A reference reflector must be identified and each trace is windowed around the reference reflection and then crosscorrelated with the trace. A normalized crosscorrelation misfit function measuring the similarity between observed and predicted crosscorrelograms is maximized for inverting the migration image. At each iteration, the weighted crosscorrelogram residual is migrated to estimate the migration image of the subsurface. The synthetic and field data tests show that ILSM can mitigate the defocusing in migration images caused by statics. Results on field data show very subtle differences between the LSM and the ILSM images because static errors in the OBS data set are not that prominent. In the synthetic examples, introduction of static errors deteriorates the LSM image, therefore we see a significant improvement in the ILSM image. However, reflectors located more than 3-4 wavelengths away from the reference reflector do not get imaged accurately. This problem can be alleviated by iteratively imaging deeper reference reflectors and using them to image even deeper areas of the subsurface. A potentially important use of ILSM is for 4D seismic surveys in which the acquisition parameters or environmental conditions can significantly change from one survey to the next. The main limitation of ILSM is that significant errors in the reference reflector's geometry can be inherited in the ILSM image. In addition, errors in the velocity model below the reference reflector can negatively impact the ILSM image. Therefore, future research should aim toward iteratively refining the estimate of the reference reflector's geometry and updating the velocity model below the reference reflector.

\section{ACKNOWLEDGMENTS}

The research reported in this publication was supported by the King Abdullah University of Science and Technology (KAUST) in Thuwal, Saudi Arabia. We thank the sponsors of the CSIM consortium for their support. We would also like to thank the high-performance computing center of KAUST for providing access to their supercomputing facilities.

\section{REFERENCES}

Bakulin, A., and R. Calvert, 2004, Virtual source: New method for imaging and 4D below complex overburden: 74th Annual International Meeting, SEG, Expanded Abstracts, 2477-2480.

Berryhill, J. R., 1986, Submarine canyons: Velocity replacement by waveequation datuming before stack: Geophysics, 51, 645-653.

Bevc, D., 1997, Flooding the topography: Wave-equation datuming of land data with rugged acquisition topography: Geophysics, 62, 1558-1569, doi: $10.1190 / 1.1444258$.

Dutta, G., M. Sinha, and S. T. Schuster, 2014, A cross-correlation objective function for least-squares migration and visco-acoustic imaging: 84th Annual International Meeting, SEG, Expanded Abstracts, 3985-3990.

Henley, D. C., 2012, Interferometric application of static corrections: Geophysics, 77, no. 1, Q1-Q13, doi: 10.1190/geo2011-0082.1.

Hornby, B. E., and J. Yu, 2007, Interferometric imaging of a salt flank using walkaway VSP data: The Leading Edge, 26, 760-763, doi: 10.1190/1 .2748493

Hornby, B. E., J. Yu, J. A. Sharp, A. Ray, Y. Quist, and C. Regone, 2006, VSP: Beyond time-to-depth: The Leading Edge, 25, 446-452, doi: 10 .1190/1.2193224.

Luo, Y., and G. Schuster, 1991, Wave equation traveltime inversion: Geophysics, 56, 645-653, doi: 10.1190/1.1443081.

Nocedal, J., and S. Wright, 2006, Numerical optimization: Springer.

Routh, P., J. Krebs, S. Lazaratos, A. Baumstein, I. Chikichev, S. Lee, N. Downey, D. Hinkley, and J. Anderson, 2011, Full-wavefield inversion of marine streamer data with the encoded simultaneous source method: 73rd Annual International Conference and Exhibition, EAGE, Extended Abstracts, F032.

Xiao, X., and G. T. Schuster, 2006, Redatuming CDP data below salt with VSP Green's function: 76th Annual International Meeting, SEG, Expanded Abstracts, 3442-3446.

Xiao, X., and G. Schuster, 2009, Local migration with extrapolated VSP Green functions: Geophysics, 74, no. 1, SI15-SI26, doi: 10.1190/1.3026619.

Yilmaz, O., and D. Lucas, 1986, Prestack layer replacement: Geophysics, 51, 1355-1369, doi: 10.1190/1.1442186.

Zhang, Y., L. Duan, and Y. Xie, 2013, A stable and practical implementation of least-squares reverse time migration: 83rd Annual International Meeting, SEG, Expanded Abstracts, 3716-3720.

Zhou, M., Z. Jiang, J. Yu, and G. Schuster, 2006, Comparison between interferometric migration and reduced-time migration of common-depthpoint data: Geophysics, 71, no. 4, SI189-SI196, doi: 10.1190/1.2213046. 